\title{
Uniqueness of Meromorphic Functions Sharing Fixed Point
}

\author{
Subhas S. Bhoosnurmath and Veena L. Pujari \\ Department of Mathematics, Karnatak University, Dharwad 580003, India \\ Correspondence should be addressed to Subhas S. Bhoosnurmath; ssbmath@gmail.com
}

Received 28 September 2012; Revised 22 January 2013; Accepted 30 January 2013

Academic Editor: Alexandre Timonov

Copyright (C) 2013 S. S. Bhoosnurmath and V. L. Pujari. This is an open access article distributed under the Creative Commons Attribution License, which permits unrestricted use, distribution, and reproduction in any medium, provided the original work is properly cited.

We study the uniqueness of meromorphic functions concerning differential polynomials sharing fixed point and obtain some significant results, which improve the results due to Lin and Yi (2004).

\section{Introduction and Main Results}

Let $f(z)$ be a nonconstant meromorphic function in the whole complex plane $\mathbb{C}$. We will use the following standard notations of value distribution theory: $T(r, f), m(r, f)$, $N(r, f), \bar{N}(r, f), \ldots$ (see $[1,2])$. We denote by $S(r, f)$ any function satisfying

$$
S(r, f)=o\{T(r, f)\}, \quad \text { as } r \longrightarrow+\infty,
$$

possibly outside of a set with finite measure.

Let $a$ be a finite complex number and $k$ a positive integer. We denote by $N_{k)}(r, 1 /(f-a))$ the counting function for the zeros of $f(z)-a$ in $|z| \leq r$ with multiplicity $\leq k$ and by $\bar{N}_{k)}(r, 1 /(f-a))$ the corresponding one for which multiplicity is not counted. Let $N_{(k}(r, 1 /(f-a))$ be the counting function for the zeros of $f(z)-a$ in $|z| \leq r$ with multiplicity $\geq k$ and $\bar{N}_{(k}(r, 1 /(f-a))$ the corresponding one for which multiplicity is not counted. Set

$$
\begin{aligned}
N_{k}\left(r, \frac{1}{f-a}\right)= & \bar{N}\left(r, \frac{1}{f-a}\right)+\bar{N}_{(2}\left(r, \frac{1}{f-a}\right) \\
& +\cdots+\bar{N}_{(k}\left(r, \frac{1}{f-a}\right) .
\end{aligned}
$$

Let $g(z)$ be a nonconstant meromorphic function. We denote by $\bar{N}_{L}(r, 1 /(f-a))$ the counting function for $a$-points of both $f(z)$ and $g(z)$ about which $f(z)$ has larger multiplicity than $g(z)$, where multiplicity is not counted. Similarly, we have notation $\bar{N}_{L}(r, 1 /(g-a))$.
We say that $f$ and $g$ share $a$ CM (counting multiplicity) if $f-a$ and $g-a$ have same zeros with the same multiplicities. Similarly, we say that $f$ and $g$ share $a$ IM (ignoring multiplicity) if $f-a$ and $g-a$ have same zeros with ignoring multiplicities.

In 2004, Lin and Yi [3] obtained the following results.

Theorem A. Let $f$ and $g$ be two transcendental meromorphic functions, $n \geq 12$ an integer. If $f^{n}(f-1) f^{\prime}$ and $g^{n}(g-1) g^{\prime}$ share $z C M$, then either $f(z) \equiv g(z)$ or

$$
g=\frac{(n+2)\left(1-h^{n+1}\right)}{(n+1)\left(1-h^{n+2}\right)}, \quad f=\frac{(n+2) h\left(1-h^{n+1}\right)}{(n+1)\left(1-h^{n+2}\right)}
$$

where $h$ is a nonconstant meromorphic function.

Theorem B. Let $f$ and $g$ be two transcendental meromorphic functions, $n \geq 13$ an integer. If $f^{n}(f-1)^{2} f^{\prime}$ and $g^{n}(g-1)^{2} g^{\prime}$ share $z C M$, then $f(z) \equiv g(z)$.

In this paper, we study the uniqueness problems of entire or meromorphic functions concerning differential polynomials sharing fixed point, which improves Theorems $\mathrm{A}$ and $\mathrm{B}$.

\subsection{Main Results}

Theorem 1. Let $f$ and $g$ be two nonconstant meromorphic functions, $n \geq 11$ a positive integer. If $f^{n}(f-1) f^{\prime}$ and 
$g^{n}(g-1) g^{\prime}$ share $z C M, f$ and $g$ share $\infty I M$, then either $f(z) \equiv g(z)$ or

$$
g=\frac{(n+2)\left(1-h^{n+1}\right)}{(n+1)\left(1-h^{n+2}\right)}, \quad f=\frac{(n+2) h\left(1-h^{n+1}\right)}{(n+1)\left(1-h^{n+2}\right)}
$$

where $h$ is a nonconstant meromorphic function.

Theorem 2. Let $f$ and $g$ be two nonconstant meromorphic functions, $n \geq 12$ a positive integer. If $f^{n}(f-1)^{2} f^{\prime}$ and $g^{n}(g-1)^{2} g^{\prime}$ share $z C M, f$ and $g$ share $\infty I M$, then $f(z) \equiv$ $g(z)$.

Theorem 3. Let $f$ and $g$ be two nonconstant entire functions, $n \geq 7$ an integer. If $f^{n}(f-1) f^{\prime}$ and $g^{n}(g-1) g^{\prime}$ share $z C M$, then $f(z) \equiv g(z)$.

\section{Some Lemmas}

Lemma 4 (see [4]). Let $f_{1}, f_{2}$, and $f_{3}$ be nonconstant meromorphic functions such that $f_{1}+f_{2}+f_{3}=1$. If $f_{1}, f_{2}$, and $f_{3}$ are linearly independent, then

$$
T\left(r, f_{1}\right)<\sum_{i=1}^{3} N_{2}\left(r, \frac{1}{f_{i}}\right)+\sum_{i=1}^{3} \bar{N}\left(r, f_{i}\right)+o(T(r))
$$

where $T(r)=\max _{1 \leq i \leq 3}\left\{T\left(r, f_{i}\right)\right\}$ and $r \notin E$.

Lemma 5 (see [1]). Let $f_{1}$ and $f_{2}$ be two nonconstant meromorphic functions. If $c_{1} f_{1}+c_{2} f_{2}=c_{3}$, where $c_{1}, c_{2}$, and $c_{3}$ are non-zero constants, then

$$
T\left(r, f_{1}\right) \leq \bar{N}\left(r, f_{1}\right)+\bar{N}\left(r, \frac{1}{f_{1}}\right)+\bar{N}\left(r, \frac{1}{f_{2}}\right)+S\left(r, f_{1}\right)
$$

Lemmas 4 and 5 play a very important role in proving our theorems.

Lemma 6 (see [1]). Let $f$ be a nonconstant meromorphic function and let $k$ be a nonnegative integer, then

$$
N\left(r, \frac{1}{f^{(k)}}\right) \leq N\left(r, \frac{1}{f}\right)+k \bar{N}(r, f)+S(r, f) .
$$

The following lemmas play a cardinal role in proving our results.

Lemma 7. Let $f$ and $g$ be nonconstant meromorphic functions. If $f^{n}(f-1) f^{\prime}$ and $g^{n}(g-1) g^{\prime}$ share $z C M$ and $n>6$, then

$$
T(r, g) \leq\left(\frac{n+3}{n-6}\right) T(r, f)+\log r+S(r, g)
$$

Proof. Applying Nevanlinna's second fundamental theorem to $g^{n}(g-1) g^{\prime}$, we have

$$
\begin{aligned}
T\left(r, g^{n}(g-1) g^{\prime}\right) \leq & \bar{N}\left(r, g^{n}(g-1) g^{\prime}\right) \\
& +\bar{N}\left(r, \frac{1}{g^{n}(g-1) g^{\prime}}\right) \\
& +\bar{N}\left(r, \frac{1}{g^{n}(g-1) g^{\prime}-z}\right)+S(r, g) \\
\leq & \bar{N}(r, g)+\bar{N}\left(r, \frac{1}{g}\right) \\
& +\bar{N}\left(r, \frac{1}{g-1}\right)+\bar{N}\left(r, \frac{1}{g^{\prime}}\right) \\
& +\bar{N}\left(r, \frac{1}{f^{n}(f-1) f^{\prime}-z}\right)+S(r, g) .
\end{aligned}
$$

By the first fundamental theorem and (9), we have

$$
\begin{aligned}
(n+1) T(r, g) \leq & T\left(r, g^{n}(g-1)\right)+S(r, g) \\
\leq & T\left(r, g^{n}(g-1) g^{\prime}\right)+T\left(r, \frac{1}{g^{\prime}}\right)+S(r, g) \\
\leq & \bar{N}(r, g)+\bar{N}\left(r, \frac{1}{g}\right) \\
& +\bar{N}\left(r, \frac{1}{g-1}\right)+\bar{N}\left(r, \frac{1}{g^{\prime}}\right) \\
& +\bar{N}\left(r, \frac{1}{f^{n}(f-1) f^{\prime}-z}\right) \\
& +T\left(r, g^{\prime}\right)+S(r, g) .
\end{aligned}
$$

We know that

$$
\begin{aligned}
\bar{N}\left(r, \frac{1}{f^{n}(f-1) f^{\prime}-z}\right) \leq & T\left(r, \frac{1}{f^{n}(f-1) f^{\prime}-z}\right) \\
= & T\left(r, f^{n}(f-1) f^{\prime}-z\right)+O(1) \\
\leq & T\left(r, f^{n}(f-1) f^{\prime}\right) \\
& +T(r, z)+O(1) \\
\leq & n T(r, f)+T(r, f-1) \\
& +T\left(r, f^{\prime}\right)+\log r+O(1) \\
\leq & (n+3) T(r, f)+\log r+O(1) .
\end{aligned}
$$


Therefore using Lemma 6, (10) becomes

$$
\begin{aligned}
(n+1) T(r, g) \leq & \bar{N}(r, g)+\bar{N}\left(r, \frac{1}{g}\right) \\
& +\bar{N}\left(r, \frac{1}{g-1}\right)+\bar{N}\left(r, \frac{1}{g^{\prime}}\right) \\
& +(n+3) T(r, f)+T\left(r, g^{\prime}\right) \\
& +\log r+S(r, g) \\
\leq & 7 T(r, g)+(n+3) T(r, f)+\log r+S(r, g)
\end{aligned}
$$

$$
\Longrightarrow(n-6) T(r, g) \leq(n+3) T(r, f)+\log r+S(r, g) \text {; }
$$

since $n>6$, we have

$$
T(r, g) \leq\left(\frac{n+3}{n-6}\right) T(r, f)+\log r+S(r, g)
$$

This completes the proof of Lemma 7.

Lemma 8. Let $f$ and $g$ be nonconstant entire functions. If $f^{n}(f-1) f^{\prime}$ and $g^{n}(g-1) g^{\prime}$ share $z C M$ and $n>3$, then

$$
T(r, g) \leq\left(\frac{n+2}{n-3}\right) T(r, f)+\log r+S(r, g) .
$$

Proof. Applying Nevanlinna's second fundamental theorem to $g^{n}(g-1) g^{\prime}$, we have

$$
\begin{aligned}
T\left(r, g^{n}(g-1) g^{\prime}\right) \leq & \bar{N}\left(r, g^{n}(g-1) g^{\prime}\right) \\
& +\bar{N}\left(r, \frac{1}{g^{n}(g-1) g^{\prime}}\right) \\
& +\bar{N}\left(r, \frac{1}{g^{n}(g-1) g^{\prime}-z}\right)+S(r, g) \\
\leq & \bar{N}(r, g)+\bar{N}\left(r, \frac{1}{g}\right) \\
& +\bar{N}\left(r, \frac{1}{g-1}\right)+\bar{N}\left(r, \frac{1}{g^{\prime}}\right) \\
& +\bar{N}\left(r, \frac{1}{f^{n}(f-1) f^{\prime}-z}\right)+S(r, g) .
\end{aligned}
$$

Since $g$ is an entire function, we have $\bar{N}(r, g)=0$ and the above equation becomes

$$
\begin{aligned}
T\left(r, g^{n}(g-1) g^{\prime}\right) \\
\leq \bar{N}\left(r, \frac{1}{g}\right)+\bar{N}\left(r, \frac{1}{g-1}\right)+\bar{N}\left(r, \frac{1}{g^{\prime}}\right) \\
\quad+\bar{N}\left(r, \frac{1}{f^{n}(f-1) f^{\prime}-z}\right)+S(r, g) .
\end{aligned}
$$

By the first fundamental theorem and (17), we have

$$
\begin{aligned}
(n+1) T(r, g) \leq & T\left(r, g^{n}(g-1)\right)+S(r, g) \\
\leq & T\left(r, g^{n}(g-1) g^{\prime}\right)+T\left(r, \frac{1}{g^{\prime}}\right)+S(r, g) \\
\leq & \bar{N}\left(r, \frac{1}{g}\right)+\bar{N}\left(r, \frac{1}{g-1}\right)+\bar{N}\left(r, \frac{1}{g^{\prime}}\right) \\
& +\bar{N}\left(r, \frac{1}{f^{n}(f-1) f^{\prime}-z}\right) \\
& +T\left(r, g^{\prime}\right)+S(r, g) .
\end{aligned}
$$

We know that

$$
\begin{aligned}
\bar{N}\left(r, \frac{1}{f^{n}(f-1) f^{\prime}-z}\right) \leq & T\left(r, \frac{1}{f^{n}(f-1) f^{\prime}-z}\right) \\
= & T\left(r, f^{n}(f-1) f^{\prime}-z\right)+O(1) \\
\leq & T\left(r, f^{n}(f-1) f^{\prime}\right) \\
& +T(r, z)+O(1) \\
\leq & n T(r, f)+T(r, f-1) \\
& +T\left(r, f^{\prime}\right)+\log r+O(1) \\
\leq & (n+2) T(r, f)+\log r+O(1) .
\end{aligned}
$$

Therefore using Lemma 6, (18) becomes

$$
\begin{aligned}
(n+1) T(r, g) \leq & \bar{N}\left(r, \frac{1}{g}\right)+\bar{N}\left(r, \frac{1}{g-1}\right)+\bar{N}\left(r, \frac{1}{g^{\prime}}\right) \\
& +(n+2) T(r, f)+T\left(r, g^{\prime}\right) \\
& +\log r+S(r, g) \\
\leq & 4 T(r, g)+(n+2) T(r, f)+\log r+S(r, g)
\end{aligned}
$$

or

$$
(n-3) T(r, g) \leq(n+2) T(r, f)+\log r+S(r, g) ;
$$

since $n>3$, we have

$$
T(r, g) \leq\left(\frac{n+2}{n-3}\right) T(r, f)+\log r+S(r, g) .
$$

This completes the proof of Lemma 8 .

Lemma 9 (see [5]). Suppose that $f(z)$ is a meromorphic function in the complex plane and $P(f)=a_{0} f^{n}+a_{1} f^{n-1}+\cdots+$ $a_{n}$, where $a_{0}(\neq 0), a_{1}, \ldots, a_{n}$ are small meromorphic functions of $f(z)$. Then

$$
T(r, P(f))=n T(r, f)+S(r, f) .
$$


Lemma 10 (see [6]). Let $f_{1}, f_{2}$, and $f_{3}$ be three meromorphic functions satisfying $\sum_{j=1}^{3} f_{j}=1$, let $g_{1}=-f_{3} / f_{2}, g_{2}=1 / f_{2}$, and $g_{3}=-f_{1} / f_{2}$. If $f_{1}, f_{2}$, and $f_{3}$ are linearly independent, then $g_{1}, g_{2}$, and $g_{3}$ are linearly independent.

\section{Proof of Theorems}

Proof of Theorem 1. By assumption, $f^{n}(f-1) f^{\prime}$ and $g^{n}(g-1) g^{\prime}$ share $z$ CM, and $f$ and $g$ share $\infty$ IM. Let

$$
H=\frac{f^{n}(f-1) f^{\prime}-z}{g^{n}(g-1) g^{\prime}-z} .
$$

Then, $H$ is a meromorphic function satisfying

$$
\begin{aligned}
T(r, H)= & T\left(r, \frac{f^{n}(f-1) f^{\prime}-z}{g^{n}(g-1) g^{\prime}-z}\right) \\
\leq & T\left(r, f^{n}(f-1) f^{\prime}-z\right) \\
& +T\left(r, g^{n}(g-1) g^{\prime}-z\right)+O(1) \\
\leq & (n+3)(T(r, f)+T(r, g))+O(\log r) .
\end{aligned}
$$

Therefore,

$$
T(r, H)=O(T(r, f)+T(r, g)) .
$$

From (24), we easily see that the zeros and poles of $H$ are multiple and satisfy

$$
\bar{N}(r, H) \leq \bar{N}_{L}(r, f), \quad \bar{N}\left(r, \frac{1}{H}\right) \leq \bar{N}_{L}(r, g) .
$$

Let

$$
\begin{gathered}
f_{1}=\frac{f^{n}(f-1) f^{\prime}}{z}, \quad f_{2}=H, \\
f_{3}=\frac{-H g^{n}(g-1) g^{\prime}}{z} .
\end{gathered}
$$

Then, $f_{1}+f_{2}+f_{3}=1$ and $T(r)$ denote the maximum of $T\left(r, f_{j}\right), j=1,2,3$.

We have

$$
\begin{gathered}
T\left(r, f_{1}\right)=O(T(r, f)), \\
T\left(r, f_{2}\right)=O(T(r, f)+T(r, g)), \\
T\left(r, f_{3}\right)=O(T(r, f)+T(r, g)) .
\end{gathered}
$$

Therefore, $T(r)=O(T(r, f)+T(r, g))$, and thus

$$
S(r, f)+S(r, g)=o(T(r)) .
$$

Now, we discuss the following three cases.

Case 1. Suppose that neither $f_{2}$ nor $f_{3}$ is a constant.
If $f_{1}, f_{2}$, and $f_{3}$ are linearly independent, then by Lemma 4 and (28), we have

$$
\begin{aligned}
T\left(r, f_{1}\right)< & \sum_{i=1}^{3} N_{2}\left(r, \frac{1}{f_{i}}\right)+\sum_{i=1}^{3} \bar{N}\left(r, f_{i}\right)+o(T(r)) \\
\leq & N_{2}\left(r, \frac{1}{f_{1}}\right)+N_{2}\left(r, \frac{1}{f_{2}}\right)+N_{2}\left(r, \frac{1}{f_{3}}\right) \\
& +\bar{N}\left(r, f_{1}\right)+\bar{N}\left(r, f_{2}\right)+\bar{N}\left(r, f_{3}\right)+o(T(r)) \\
\leq & N_{2}\left(r, \frac{z}{f^{n}(f-1) f^{\prime}}\right)+N_{2}\left(r, \frac{1}{H}\right) \\
& +N_{2}\left(r, \frac{z}{H g^{n}(g-1) g^{\prime}}\right) \\
& +\bar{N}\left(r, \frac{f^{n}(f-1) f^{\prime}}{z}\right) \\
& +\bar{N}(r, H)+\bar{N}\left(r, \frac{H g^{n}(g-1) g^{\prime}}{z}\right)+o(T(r)) \\
= & N_{2}\left(r, \frac{1}{f^{n}(f-1) f^{\prime}}\right)+N_{2}\left(r, \frac{1}{H}\right) \\
& +N_{2}\left(r, \frac{1}{H g^{n}(g-1) g^{\prime}}\right) \\
& +\bar{N}\left(r, f^{n}(f-1) f^{\prime}\right) \\
& +\bar{N}(r, H)+\bar{N}\left(r, H g^{n}(g-1) g^{\prime}\right) \\
& +2 \log r+o(T(r)) .
\end{aligned}
$$

Using (27), we note that

$$
\begin{aligned}
N_{2}\left(r, \frac{1}{H g^{n}(g-1) g^{\prime}}\right) \\
\quad \leq N_{2}\left(r, \frac{1}{H}\right)+N_{2}\left(r, \frac{1}{g^{n}(g-1) g^{\prime}}\right) \\
\quad \leq 2 \bar{N}\left(r, \frac{1}{H}\right)+N_{2}\left(r, \frac{1}{g^{n}(g-1) g^{\prime}}\right) \\
\quad \leq 2 \bar{N}_{L}(r, g)+N_{2}\left(r, \frac{1}{g^{n}(g-1) g^{\prime}}\right) .
\end{aligned}
$$

Since $\bar{N}_{L}(r, g)=0$, we obtain that

$$
\begin{aligned}
N_{2}\left(r, \frac{1}{H g^{n}(g-1) g^{\prime}}\right) & \leq N_{2}\left(r, \frac{1}{g^{n}(g-1) g^{\prime}}\right), \\
\bar{N}\left(r, H g^{n}(g-1) g^{\prime}\right) & \leq \bar{N}(r, H)+\bar{N}\left(r, g^{n}(g-1) g^{\prime}\right) \\
& \leq \bar{N}_{L}(r, f)+\bar{N}(r, g) .
\end{aligned}
$$


But $\bar{N}_{L}(r, f)=0$, so we get

$$
\bar{N}\left(r, H g^{n}(g-1) g^{\prime}\right) \leq \bar{N}(r, g) .
$$

Using (33) and (35) in (31), we get

$$
\begin{aligned}
T\left(r, f_{1}\right) \leq & N_{2}\left(r, \frac{1}{f^{n}(f-1) f^{\prime}}\right)+N_{2}\left(r, \frac{1}{H}\right) \\
& +N_{2}\left(r, \frac{1}{g^{n}(g-1) g^{\prime}}\right)+\bar{N}(r, f) \\
& +\bar{N}(r, H)+\bar{N}(r, g)+2 \log r+o(T(r)) .
\end{aligned}
$$

Since $f$ and $g$ share $\infty$ IM, we have $\bar{N}(r, f)=\bar{N}(r, g)$.

Using this with (27), we get

$$
\begin{aligned}
& T\left(r, f_{1}\right) \leq N_{2}\left(r, \frac{1}{f^{n}(f-1) f^{\prime}}\right)+2 \bar{N}_{L}(r, g) \\
& +N_{2}\left(r, \frac{1}{g^{n}(g-1) g^{\prime}}\right)+\bar{N}_{L}(r, f) \\
& +2 \bar{N}(r, f)+2 \log r+o(T(r)) \\
& \leq N\left(r, \frac{1}{f^{n}(f-1) f^{\prime}}\right) \\
& -\left[N_{(3}\left(r, \frac{1}{f^{n}(f-1) f^{\prime}}\right)\right. \\
& \left.-2 \bar{N}_{(3}\left(r, \frac{1}{f^{n}(f-1) f^{\prime}}\right)\right] \\
& +N\left(r, \frac{1}{g^{n}(g-1) g^{\prime}}\right) \\
& -\left[N_{(3}\left(r, \frac{1}{g^{n}(g-1) g^{\prime}}\right)\right. \\
& \left.-2 \bar{N}_{(3}\left(r, \frac{1}{g^{n}(g-1) g^{\prime}}\right)\right] \\
& +2 \bar{N}_{L}(r, g)+\bar{N}_{L}(r, f)+2 \bar{N}(r, f) \\
& +2 \log r+o(T(r)) \text {. }
\end{aligned}
$$

If $z_{0}$ is a zero of $f$ with multiplicity $p$, then $z_{0}$ is a zero of $f^{n}(f-1) f^{\prime}$ with multiplicity $n p+p-1 \geq 3$; we have

$$
\begin{aligned}
& {\left[N_{(3}\left(r, \frac{1}{f^{n}(f-1) f^{\prime}}\right)-2 \bar{N}_{(3}\left(r, \frac{1}{f^{n}(f-1) f^{\prime}}\right)\right]} \\
& \quad \geq(n-2) N\left(r, \frac{1}{f}\right) .
\end{aligned}
$$

Similarly,

$$
\begin{aligned}
& {\left[N_{(3}\left(r, \frac{1}{g^{n}(g-1) g^{\prime}}\right)-2 \bar{N}_{(3}\left(r, \frac{1}{g^{n}(g-1) g^{\prime}}\right)\right]} \\
& \quad \geq(n-2) N\left(r, \frac{1}{g}\right) .
\end{aligned}
$$

Let

$$
f_{1}^{*}=\frac{f^{n+2}}{n+2}-\frac{f^{n+1}}{n+1} \text {. }
$$

By Lemma 9, we have $T\left(r, f_{1}^{*}\right)=(n+2) T(r, f)+S(r, f)$.

Since $\left(f_{1}^{*}\right)^{\prime}=z f_{1}$, we have

$$
\begin{aligned}
m\left(r, \frac{1}{f_{1}^{*}}\right) & \leq m\left(r, \frac{1}{z f_{1}}\right)+m\left(r, \frac{\left(f_{1}^{*}\right)^{\prime}}{f_{1}^{*}}\right) \\
& \leq m\left(r, \frac{1}{f_{1}}\right)+\log r+S(r, f) .
\end{aligned}
$$

By the first fundamental theorem, we have

$$
\begin{aligned}
T\left(r, f_{1}^{*}\right) \leq & T\left(r, f_{1}\right)+N\left(r, \frac{1}{f_{1}^{*}}\right)-N\left(r, \frac{1}{f_{1}}\right) \\
& +\log r+S(r, f) ;
\end{aligned}
$$

we have

$$
\begin{aligned}
& N\left(r, \frac{1}{f_{1}^{*}}\right) \\
& \quad=(n+1) N\left(r, \frac{1}{f}\right)+N\left(r, \frac{1}{f-(n+2) /(n+1)}\right) .
\end{aligned}
$$

From (37)-(43), we get

$$
\begin{aligned}
T\left(r, f_{1}^{*}\right) \leq & N\left(r, \frac{1}{f^{n}(f-1) f^{\prime}}\right)-(n-2) N\left(r, \frac{1}{f}\right) \\
& +N\left(r, \frac{1}{g^{n}(g-1) g^{\prime}}\right)-(n-2) N\left(r, \frac{1}{g}\right) \\
& +2 \bar{N}_{L}(r, g) \\
& +\bar{N}_{L}(r, f)+2 \bar{N}(r, f)+(n+1) N\left(r, \frac{1}{f}\right) \\
& +N\left(r, \frac{1}{f-(n+2) /(n+1)}\right) \\
& -N\left(r, \frac{1}{f^{n}(f-1) f^{\prime}}\right)+3 \log r+o(T(r)) .
\end{aligned}
$$


Using Lemma 6, we get

$$
\begin{aligned}
T\left(r, f_{1}^{*}\right) \leq & 3 N\left(r, \frac{1}{f}\right)+3 N\left(r, \frac{1}{g}\right) \\
& +2 \bar{N}(r, f)+\bar{N}(r, g)+2 \bar{N}_{L}(r, g) \\
& +\bar{N}_{L}(r, f)+N\left(r, \frac{1}{g-1}\right) \\
& +N(r, \overline{f-(n+2) /(n+1)}) \\
& +3 \log r+o(T(r)) \\
(n+2) T(r, f) \leq & 3 N\left(r, \frac{1}{f}\right)+3 N\left(r, \frac{1}{g}\right) \\
& +2 \bar{N}_{(r, f)+\bar{N}(r, g)} \\
& +2 \bar{N}_{L}(r, g)+\bar{N}_{L}(r, f)+N\left(r, \frac{1}{g-1}\right) \\
& +N\left(r, \frac{1}{f-(n+2) /(n+1)}\right) \\
& +3 \log r+o(T(r)) . \\
& \\
& \\
&
\end{aligned}
$$

Let

$$
\begin{gathered}
g_{1}=-\frac{f_{3}}{f_{2}}=\frac{g^{n}(g-1) g^{\prime}}{z}, \\
g_{2}=\frac{1}{f_{2}}=\frac{1}{H}, \quad g_{3}=-\frac{f_{1}}{f_{2}}=\frac{f^{n}(f-1) f^{\prime}}{z H} .
\end{gathered}
$$

Then $g_{1}+g_{2}+g_{3}=1$. By Lemma $10, g_{1}, g_{2}$, and $g_{3}$ are linearly independent. In the same manner as above, we get

$$
\begin{aligned}
(n+2) T(r, g) \leq & 3 N\left(r, \frac{1}{g}\right)+3 N\left(r, \frac{1}{f}\right) \\
& +2 \bar{N}(r, g)+\bar{N}(r, f)+2 \bar{N}_{L}(r, f) \\
& +\bar{N}_{L}(r, g)+N\left(r, \frac{1}{f-1}\right) \\
& +N\left(r, \frac{1}{g-(n+2) /(n+1)}\right) \\
& +3 \log r+o(T(r)) .
\end{aligned}
$$

Note that

$$
\bar{N}_{L}(r, f)+\bar{N}_{L}(r, g) \leq \bar{N}(r, f)=\bar{N}(r, g) .
$$

Adding (45) and (47) gives

$$
\begin{aligned}
(n+2) & (T(r, f)+T(r, g)) \\
\leq & 6\left(N\left(r, \frac{1}{f}\right)+N\left(r, \frac{1}{g}\right)\right)+3(\bar{N}(r, f)+\bar{N}(r, g)) \\
& +\left(\bar{N}_{L}(r, f)+\bar{N}_{L}(r, g)\right) \\
& +\left(N\left(r, \frac{1}{f-1}\right)+N\left(r, \frac{1}{g-1}\right)\right) \\
& +N\left(r, \frac{1}{f-(n+2) /(n+1)}\right) \\
& +N\left(r, \frac{1}{g-(n+2) /(n+1)}\right)+6 \log r+o(T(r)) \\
(n-6) & (T(r, f)+T(r, g)) \\
\leq & 3\left(\bar{N}_{(r, f)+\bar{N}(r, g))}\right. \\
& +3\left(\bar{N}_{L}(r, f)+\bar{N}_{L}(r, g)\right)+6 \log r+o(T(r)) .
\end{aligned}
$$

Using (48), we get

$$
\begin{aligned}
(n-6) & (T(r, f)+T(r, g)) \\
\leq & 3 \bar{N}(r, f)+6 \bar{N}(r, g)+6 \log r+o(T(r))
\end{aligned}
$$

or

$$
\begin{aligned}
(n-6) & (T(r, f)+T(r, g)) \\
\leq & 6 \bar{N}(r, f)+3 \bar{N}(r, g)+6 \log r+o(T(r)) .
\end{aligned}
$$

Combining (50) and (51), we get

$$
\begin{aligned}
(n-6)(T(r, f)+T(r, g)) \leq & \frac{9}{2}(\bar{N}(r, f)+\bar{N}(r, g)) \\
& +6 \log r+o(T(r)) \\
\left(n-\frac{21}{2}\right)(T(r, f)+T(r, g)) & \leq 6 \log r+o(T(r)) .
\end{aligned}
$$

By $n \geq 11$ and (30), we get a contradiction. Thus $f_{1}, f_{2}$, and $f_{3}$ are linearly dependent. Then, there exists three constants $\left(c_{1}, c_{2}, c_{3}\right) \neq(0,0,0)$ such that

$$
c_{1} f_{1}+c_{2} f_{2}+c_{3} f_{3}=0 .
$$

If $c_{1}=0$, from $(53) c_{2} \neq 0, c_{3} \neq 0$, and

$$
f_{3}=-\frac{c_{2}}{c_{3}} f_{2}
$$

$$
\Longrightarrow g^{n}(g-1) g^{\prime}=\frac{c_{2}}{c_{3}} z \text {. }
$$


On integrating, we get

$$
\begin{gathered}
\frac{g^{n+2}}{n+2}-\frac{g^{n+1}}{n+1}=\frac{c_{2}}{c_{3}} \frac{z^{2}}{2}+k, \quad k \text { is a constant, } \\
T\left(r, \frac{g^{n+2}}{n+2}-\frac{g^{n+1}}{n+1}\right) \leq T\left(r, z^{2}\right)+O(1) \\
(n+2) T(r, g) \leq 2 \log r+O(1)
\end{gathered}
$$

since $n \geq 11$, we get a contradiction.

Thus $c_{1} \neq 0$, and by (53) we have

$$
\begin{aligned}
c_{1} f_{1} & =-c_{2} f_{2}-c_{3} f_{3} \\
\Longrightarrow f_{1} & =-\frac{c_{2}}{c_{1}} f_{2}-\frac{c_{3}}{c_{1}} f_{3} .
\end{aligned}
$$

Substituting this in $f_{1}+f_{2}+f_{3}=1$, we get

$$
-\frac{c_{2}}{c_{1}} f_{2}-\frac{c_{3}}{c_{1}} f_{3}+f_{2}+f_{3}=1
$$

that is,

$$
\left(1-\frac{c_{2}}{c_{1}}\right) f_{2}+\left(1-\frac{c_{3}}{c_{1}}\right) f_{3}=1, \quad \text { where } c_{1} \neq c_{3}, c_{2} \neq c_{3} \text {. }
$$

From (28), we obtain

$$
\begin{aligned}
& \left(1-\frac{c_{2}}{c_{1}}\right) H+\left(1-\frac{c_{3}}{c_{1}}\right)\left(\frac{-H g^{n}(g-1) g^{\prime}}{z}\right)=1 \\
& \Longrightarrow\left(1-\frac{c_{2}}{c_{1}}\right)-\left(1-\frac{c_{3}}{c_{1}}\right)\left(\frac{g^{n}(g-1) g^{\prime}}{z}\right)=1 \\
& \Longrightarrow\left(1-\frac{c_{3}}{c_{1}}\right)\left(\frac{g^{n}(g-1) g^{\prime}}{z}\right)+\frac{1}{H}=\left(1-\frac{c_{2}}{c_{1}}\right) .
\end{aligned}
$$

Note that

$$
\begin{aligned}
T\left(r, g^{n}(g-1) g^{\prime}\right) & \leq T\left(r, \frac{g^{n}(g-1) g^{\prime}}{z}\right)+T(r, z) \\
& \leq T\left(r, \frac{g^{n}(g-1) g^{\prime}}{z}\right)+\log r
\end{aligned}
$$

Using (61), we get

$$
\begin{aligned}
T\left(r, g^{n}(g-1) g^{\prime}\right) \leq & \bar{N}(r, g)+\bar{N}\left(r, \frac{1}{g^{n}(g-1) g^{\prime}}\right) \\
& +\bar{N}(r, g)+2 \log r+S(r, g) \\
= & \bar{N}\left(r, \frac{1}{g^{n}(g-1) g^{\prime}}\right)+2 \bar{N}(r, g) \\
& +2 \log r+S(r, g) .
\end{aligned}
$$

By Lemmas 9 and 6 and (63), we have

$$
\begin{aligned}
(n+1) T(r, g)= & T\left(r, g^{n}(g-1)\right)+S(r, g) \\
\leq & T\left(r, g^{n}(g-1) g^{\prime}\right)+T\left(r, \frac{1}{g^{\prime}}\right)+S(r, g) \\
\leq & \bar{N}\left(r, \frac{1}{g^{n}(g-1) g^{\prime}}\right)+2 \bar{N}(r, g) \\
& +2 \log r+2 T(r, g)+S(r, g) \\
\leq & \bar{N}\left(r, \frac{1}{g}\right)+\bar{N}\left(r, \frac{1}{g-1}\right)+N\left(r, \frac{1}{g^{\prime}}\right) \\
& +2 \bar{N}(r, g)+2 T(r, g)+2 \log r+S(r, g) \\
\leq & 8 T(r, g)+2 \log r+S(r, g) \\
\Longrightarrow & (n-7) T(r, g) \leq 2 \log r+S(r, g) ;
\end{aligned}
$$

we obtain $n \leq 7$, which contradicts $n \geq 11$.

Case 2. Suppose that $f_{2}=c(\neq 0)$, where $c$ is a constant. If $c \neq 1$, then we have

$$
\begin{gathered}
f_{1}+f_{2}+f_{3}=1 \\
\Longrightarrow \frac{f^{n}(f-1) f^{\prime}}{z}+c-\frac{c g^{n}(g-1) g^{\prime}}{z}=1 ; \\
\Longrightarrow \frac{f^{n}(f-1) f^{\prime}}{z}-\frac{c g^{n}(g-1) g^{\prime}}{z}=1-c .
\end{gathered}
$$

Applying Lemma 5 to the above equation, we have

$$
\begin{aligned}
T\left(r, \frac{f^{n}(f-1) f^{\prime}}{z}\right) \leq & \bar{N}\left(r, \frac{f^{n}(f-1) f^{\prime}}{z}\right) \\
& +\bar{N}\left(r, \frac{z}{g^{n}(g-1) g^{\prime}}\right) \\
& +\bar{N}\left(r, \frac{z}{f^{n}(f-1) f^{\prime}}\right)+S(r, f)
\end{aligned}
$$




$$
\begin{aligned}
\leq & \bar{N}(r, f)+\bar{N}\left(r, \frac{1}{g^{n}(g-1) g^{\prime}}\right) \\
& +\bar{N}\left(r, \frac{1}{f^{n}(f-1) f^{\prime}}\right) \\
& +\log r+S(r, f) .
\end{aligned}
$$

Note that

$$
T\left(r, f^{n}(f-1) f^{\prime}\right) \leq T\left(r, \frac{f^{n}(f-1) f^{\prime}}{z}\right)+\log r .
$$

Therefore,

$$
\begin{aligned}
T\left(r, f^{n}(f-1) f^{\prime}\right) \leq & \bar{N}(r, f)+\bar{N}\left(r, \frac{1}{g^{n}(g-1) g^{\prime}}\right) \\
& +\bar{N}\left(r, \frac{1}{f^{n}(f-1) f^{\prime}}\right) \\
& +2 \log r+S(r, f) .
\end{aligned}
$$

Using Lemmas 9 and 6 and (69), we have

$$
\begin{aligned}
(n+1) T(r, f)= & T\left(r, f^{n}(f-1)\right)+S(r, f) \\
\leq & T\left(r, f^{n}(f-1) f^{\prime}\right) \\
& +T\left(r, \frac{1}{f^{\prime}}\right)+S(r, f) \\
\leq & \bar{N}(r, f)+\bar{N}\left(r, \frac{1}{f^{n}(f-1) f^{\prime}}\right) \\
& +\bar{N}\left(r, \frac{1}{g^{n}(g-1) g^{\prime}}\right) \\
& +T\left(r, f^{\prime}\right)+2 \log r+S(r, f) \\
\leq & \bar{N}(r, f)+\bar{N}\left(r, \frac{1}{f}\right) \\
& +\bar{N}\left(r, \frac{1}{f-1}\right)+N\left(r, \frac{1}{f^{\prime}}\right)
\end{aligned}
$$

$$
\begin{aligned}
&+\bar{N}\left(r, \frac{1}{g}\right)+\bar{N}\left(r, \frac{1}{g-1}\right) \\
&+N\left(r, \frac{1}{g^{\prime}}\right)+2 T(r, f) \\
&+2 \log r+S(r, f) \\
& \leq 7 T(r, f)+4 T(r, g)+2 \log r+S(r, f) \\
& \Longrightarrow(n-6) T(r, f) \leq 4 T(r, g)+2 \log r+S(r, f) .
\end{aligned}
$$

Using Lemma 7, we get

$$
(n-6) T(r, f) \leq 4\left(\frac{n+3}{n-6}\right) T(r, f)+2 \log r+S(r, f)
$$

since $n \geq 11$, we get a contradiction.

Therefore $c=1$, and by (27) and (24) we have

$$
f^{n}(f-1) f^{\prime}=g^{n}(g-1) g^{\prime} .
$$

On integrating, we get

$$
\begin{gathered}
\frac{f^{n+2}}{n+2}-\frac{f^{n+1}}{n+1}=\frac{g^{n+2}}{n+2}-\frac{g^{n+1}}{n+1}+k \\
F^{*}=G^{*}+k, \quad \text { where } k \text { is a constant. }
\end{gathered}
$$

We claim that $k=0$. Suppose that $k \neq 0$, then

$$
\begin{aligned}
& \Theta\left(0, F^{*}\right)+\Theta\left(k, F^{*}\right)+\Theta\left(\infty, F^{*}\right) \\
& =\Theta\left(0, F^{*}\right)+\Theta\left(0, G^{*}\right)+\Theta\left(\infty, F^{*}\right) .
\end{aligned}
$$

We have

$$
\begin{aligned}
\bar{N}\left(r, \frac{1}{F^{*}}\right) \\
\quad=\bar{N}\left(r, \frac{1}{f}\right)+\bar{N}\left(r, \frac{1}{f-(n+2) /(n+1)}\right) \leq 2 T(r, f) .
\end{aligned}
$$

Similarly,

$$
\begin{gathered}
\bar{N}\left(r, \frac{1}{G^{*}}\right) \leq 2 T(r, g), \\
\bar{N}\left(r, F^{*}\right)=\bar{N}(r, f) \leq T(r, f) .
\end{gathered}
$$

Using Lemma 9, we have

$$
\begin{aligned}
& T\left(r, F^{*}\right)=(n+2) T(r, f)+S(r, f), \\
& T\left(r, G^{*}\right)=(n+2) T(r, g)+S(r, g) .
\end{aligned}
$$

Thus,

$$
\Theta\left(0, F^{*}\right)=1-\varlimsup_{r \rightarrow \infty} \frac{\bar{N}\left(r, 1 / F^{*}\right)}{T\left(r, F^{*}\right)} \geq 1-\frac{2}{n+2} .
$$


Similarly,

$$
\begin{gathered}
\Theta\left(0, G^{*}\right) \geq 1-\frac{2}{n+2}, \\
\Theta\left(\infty, F^{*}\right)=1-\varlimsup_{r \rightarrow \infty} \frac{\bar{N}\left(r, F^{*}\right)}{T\left(r, F^{*}\right)} \geq 1-\frac{1}{n+2} .
\end{gathered}
$$

Therefore, (74) becomes

$$
\begin{array}{r}
\Theta\left(0, F^{*}\right)+\Theta\left(k, F^{*}\right)+\Theta\left(\infty, F^{*}\right) \\
\geq 2\left(1-\frac{2}{n+2}\right)+1-\frac{1}{n+2} \\
=\frac{3 n+1}{n+2}>2 \quad \text { for } n \geq 11,
\end{array}
$$

which contradicts $\sum_{a \in \overline{\mathscr{C}}} \Theta(a, f) \leq 2$. Thus, we have

$$
\frac{f^{n+2}}{n+2}-\frac{f^{n+1}}{n+1}=\frac{g^{n+2}}{n+2}-\frac{g^{n+1}}{n+1} .
$$

Let $h=f / g$. If $h \neq \equiv 1$, then we easily obtain that

$$
g=\frac{(n+2)\left(h^{n+1}-1\right)}{(n+1)\left(h^{n+2}-1\right)}, \quad f=\frac{(n+2) h\left(h^{n+1}-1\right)}{(n+1)\left(h^{n+2}-1\right)} .
$$

If $h \equiv 1$, that is, $f \equiv g$.

Case 3. Suppose that $f_{3}=c(\neq 0)$, where $c$ is a constant.

If $c \neq 1$, then we have

$$
\begin{gathered}
f_{1}+f_{2}+f_{3}=1 \\
f_{1}+f_{2}=1-c \\
\frac{f^{n}(f-1) f^{\prime}}{z}-\frac{c z}{g^{n}(g-1) g^{\prime}}=1-c .
\end{gathered}
$$

Applying Lemma 5 to the above equation, we have

$$
\begin{aligned}
T\left(r, \frac{f^{n}(f-1) f^{\prime}}{z}\right) \leq & \bar{N}\left(r, \frac{f^{n}(f-1) f^{\prime}}{z}\right) \\
& +\bar{N}\left(r, \frac{z}{f^{n}(f-1) f^{\prime}}\right) \\
& +\bar{N}\left(r, \frac{g^{n}(g-1) g^{\prime}}{z}\right)+S(r, f) \\
\leq & \bar{N}(r, f)+\bar{N}\left(r, \frac{1}{f^{n}(f-1) f^{\prime}}\right) \\
& +\bar{N}(r, g)+2 \log r+S(r, f) .
\end{aligned}
$$

Note that

$$
\begin{aligned}
T\left(r, f^{n}(f-1) f^{\prime}\right) & \leq T\left(r, \frac{f^{n}(f-1) f^{\prime}}{z}\right)+T(r, z) \\
& \leq T\left(r, \frac{f^{n}(f-1) f^{\prime}}{z}\right)+\log r .
\end{aligned}
$$

Therefore using (84), we have

$$
\begin{aligned}
T\left(r, f^{n}(f-1) f^{\prime}\right) \leq & \bar{N}(r, f)+\bar{N}\left(r, \frac{1}{f^{n}(f-1) f^{\prime}}\right) \\
& +\bar{N}(r, g)+3 \log r+S(r, f) .
\end{aligned}
$$

Using Lemmas 9 and 6 and (86), we have

$$
\begin{aligned}
(n+1) T(r, f)= & T\left(r, f^{n}(f-1)\right)+S(r, f) \\
\leq & T\left(r, f^{n}(f-1) f^{\prime}\right) \\
& +T\left(r, \frac{1}{f^{\prime}}\right)+S(r, f) \\
\leq & \bar{N}(r, f)+\bar{N}\left(r, \frac{1}{f^{n}(f-1) f^{\prime}}\right) \\
& +\bar{N}(r, g)+T\left(r, f^{\prime}\right)+3 \log r+S(r, f) \\
\leq & \bar{N}(r, f)+\bar{N}\left(r, \frac{1}{f}\right)+\bar{N}\left(r, \frac{1}{f-1}\right) \\
& +N\left(r, \frac{1}{f^{\prime}}\right)+\bar{N}(r, g)+2 T(r, f) \\
& +3 \log r+S(r, f) \\
\leq & 7 T(r, f)+T(r, g)+3 \log r+S(r, f) \\
\Longrightarrow(n-6) T & (r, f) \leq T(r, g)+3 \log r+S(r, f) .
\end{aligned}
$$

Using Lemma 7, we get

$$
(n-6) T(r, f) \leq \frac{(n+3)}{(n-6)} T(r, f)+3 \log r+S(r, f)
$$

since $n \geq 11$, we get a contradiction. Thus, $c=1$. Hence,

$$
\begin{gathered}
\frac{f^{n}(f-1) f^{\prime}}{z}-\frac{z}{g^{n}(g-1) g^{\prime}}=0 \\
\Longrightarrow f^{n}(f-1) f^{\prime} g^{n}(g-1) g^{\prime}=z^{2} .
\end{gathered}
$$

Let $z_{0}$ be a zero of $f$ of order $p$. From (89), we know that $z_{0}$ is a pole of $g$. Suppose that $z_{0}$ is a pole of $g$ of order $q$. From (89), we obtain

$$
\begin{aligned}
& n p+p-1=n q+2 q+1 \\
& \Longrightarrow(n+1)(p-q)=q+2,
\end{aligned}
$$

$$
p \geq n
$$


Let $z_{1}$ be a zero of $(f-1)$ of order $p_{1}$, then from $(89) z_{1}$ is a pole of $g$ (say order $q_{1}$ ). By (89), we get

$$
\begin{gathered}
p_{1}+p_{1}-1=n q_{1}+2 q_{1}+1 \\
2 p_{1}-1 \geq n+3 \\
2 p_{1} \geq n+4 \Longrightarrow p_{1} \geq \frac{n+4}{2} .
\end{gathered}
$$

Let $z_{2}$ be a zero of $f^{\prime}$ of order $p_{2}$ that is not zero of $f(f-1)$, then from (89), $z_{2}$ is a pole of $g$ of order $q_{2}$. Again by (89), we get

$$
\begin{gathered}
p_{2}=n q_{2}+2 q_{2}+1 \\
\Longrightarrow p_{2} \geq n+3
\end{gathered}
$$

In the same manner as above, we have similar results for the zeros of $g^{n}(g-1) g^{\prime}$. From (89)-(93), we have

$$
\bar{N}\left(r, f^{n}(f-1) f^{\prime}\right)=\bar{N}\left(r, \frac{z^{2}}{g^{n}(g-1) g^{\prime}}\right) ;
$$

that is,

$$
\begin{aligned}
\bar{N}(r, f) & \leq \bar{N}\left(r, \frac{1}{g}\right)+\bar{N}\left(r, \frac{1}{g-1}\right)+N\left(r, \frac{1}{g^{\prime}}\right) \\
& \leq \frac{1}{11} N\left(r, \frac{1}{g}\right)+\frac{2}{15} N\left(r, \frac{1}{g-1}\right)+\frac{1}{14} N\left(r, \frac{1}{g^{\prime}}\right) \\
& \leq \frac{1}{11} T(r, g)+\frac{2}{15} T(r, g)+\frac{2}{14} T(r, g)+s(r, g) \\
& =\left(\frac{1}{11}+\frac{2}{15}+\frac{2}{7}\right) T(r, g)+S(r, g) \\
& \Longrightarrow \bar{N}(r, f)<\frac{2}{3} T(r, g)+S(r, g)
\end{aligned}
$$

By Nevanlinna's second fundamental theorem, we have from (91), (92), and (95) that

$$
\begin{aligned}
T(r, f) \leq & \bar{N}\left(r, \frac{1}{f}\right)+\bar{N}\left(r, \frac{1}{f-1}\right)+\bar{N}(r, f)+S(r, f) \\
< & \frac{1}{11} T(r, f)+\frac{2}{15} T(r, f)+\frac{2}{3} T(r, g) \\
& +S(r, f)+S(r, g) \\
& \Longrightarrow T(r, f) \\
& \leq \frac{37}{165} T(r, f)+\frac{2}{3} T(r, g)+S(r, f)+S(r, g) .
\end{aligned}
$$

Similarly,

$$
T(r, g) \leq \frac{37}{165} T(r, g)+\frac{2}{3} T(r, f)+S(r, f)+S(r, g) .
$$

From (96) and (97), we get

$$
\begin{aligned}
& T(r, f)+T(r, g) \\
& \leq \frac{37}{165}(T(r, f)+T(r, g)) \\
&+\frac{2}{3}(T(r, f)+T(r, g))+S(r, f)+S(r, g) \\
& \frac{18}{165}(T(r, f)+T(r, g)) \leq S(r, f)+S(r, g) ;
\end{aligned}
$$

since $n \geq 11$, we get a contradiction.

This completes the proof of Theorem 1.

Using the same argument as in the proof of Theorem 1, we can prove Theorem 2 .

Proof of Theorem 3. By the assumption of the theorem, we know that either both $f$ and $g$ are two transcendental entire functions or both $f$ and $g$ are polynomials.

If $f$ and $g$ are transcendental entire functions, putting $\bar{N}(r, f)=0, \bar{N}(r, g)=0$ and using similar arguments as in the proof of Theorem 1, we easily obtain Theorem 3 .

If $f$ and $g$ are polynomials, $f^{n}(f-1) f^{\prime}$ and $g^{n}(g-1) g^{\prime}$ share $z \mathrm{CM}$, we get

$$
f^{n}(f-1) f^{\prime}-z=k\left(g^{n}(g-1) g^{\prime}-z\right),
$$

where $k$ is a nonzero constant. Suppose that $k \neq 1$, (99) can be written as

$$
\frac{f^{n}(f-1) f^{\prime}}{z}-k \frac{g^{n}(g-1) g^{\prime}}{z}=1-k .
$$

Applying Lemma 5 to the above equation, we have

$$
\begin{aligned}
T\left(r, \frac{f^{n}(f-1) f^{\prime}}{z}\right) \leq & \bar{N}\left(r, \frac{f^{n}(f-1) f^{\prime}}{z}\right) \\
& +\bar{N}\left(r, \frac{z}{g^{n}(g-1) g^{\prime}}\right) \\
& +\bar{N}\left(r, \frac{z}{f^{n}(f-1) f^{\prime}}\right)+S(r, f) .
\end{aligned}
$$

Since $f$ is a polynomial, so it does not have any poles. Thus, we have,

$$
\begin{aligned}
T(r, & \left.\frac{f^{n}(f-1) f^{\prime}}{z}\right) \\
\leq & \bar{N}\left(r, \frac{1}{g^{n}(g-1) g^{\prime}}\right) \\
& +\bar{N}\left(r, \frac{1}{f^{n}(f-1) f^{\prime}}\right)+2 \log r+S(r, f) .
\end{aligned}
$$

Note that

$$
T\left(r, f^{n}(f-1) f^{\prime}\right) \leq T\left(r, \frac{f^{n}(f-1) f^{\prime}}{z}\right)+\log r .
$$


Therefore,

$$
\begin{aligned}
T\left(r, f^{n}\right. & \left.(f-1) f^{\prime}\right) \\
\leq & \bar{N}\left(r, \frac{1}{g^{n}(g-1) g^{\prime}}\right) \\
& +\bar{N}\left(r, \frac{1}{f^{n}(f-1) f^{\prime}}\right)+3 \log r+S(r, f) .
\end{aligned}
$$

Using Lemmas 9 and 6 and (104), we have

$$
\begin{aligned}
& (n+1) T(r, f)=T\left(r, f^{n}(f-1)\right)+S(r, f) \\
& \leq T\left(r, f^{n}(f-1) f^{\prime}\right) \\
& +T\left(r, \frac{1}{f^{\prime}}\right)+S(r, f) \\
& \leq \bar{N}\left(r, \frac{1}{f^{n}(f-1) f^{\prime}}\right) \\
& +\bar{N}\left(r, \frac{1}{g^{n}(g-1) g^{\prime}}\right) \\
& +T\left(r, f^{\prime}\right)+3 \log r+S(r, f) \\
& \leq \bar{N}\left(r, \frac{1}{f}\right)+\bar{N}\left(r, \frac{1}{f-1}\right)+N\left(r, \frac{1}{f^{\prime}}\right) \\
& +\bar{N}\left(r, \frac{1}{g}\right)+\bar{N}\left(r, \frac{1}{g-1}\right) \\
& +N\left(r, \frac{1}{g^{\prime}}\right)+T(r, f) \\
& +3 \log r+S(r, f) \\
& \leq 4 T(r, f)+3 T(r, g)+3 \log r+S(r, f) \\
& \Longrightarrow(n-3) T(r, f) \leq 3 T(r, g)+3 \log r+S(r, f) \text {. }
\end{aligned}
$$

Using Lemma 8, we get

$$
(n-3) T(r, f) \leq 3\left(\frac{n+2}{n-3}\right) T(r, f)+3 \log r+S(r, f) \text {; }
$$

since $n \geq 7$, we get a contradiction.

Therefore, $k=1$; so (99) becomes

$$
f^{n}(f-1) f^{\prime}=g^{n}(g-1) g^{\prime} .
$$

On integrating, we get

$$
\begin{gathered}
\frac{f^{n+2}}{n+2}-\frac{f^{n+1}}{n+1}=\frac{g^{n+2}}{n+2}-\frac{g^{n+1}}{n+1}+c \\
F^{*}=G^{*}+c, \quad \text { where } c \text { is a constant. }
\end{gathered}
$$

We claim that $c=0$. Suppose that $c \neq 0$, then

$$
\Theta\left(0, F^{*}\right)+\Theta\left(c, F^{*}\right)=\Theta\left(0, F^{*}\right)+\Theta\left(0, G^{*}\right) .
$$

We have

$$
\begin{aligned}
& \bar{N}\left(r, \frac{1}{F^{*}}\right) \\
& \quad=\bar{N}\left(r, \frac{1}{f}\right)+\bar{N}\left(r, \frac{1}{f-(n+2) /(n+1)}\right) \leq 2 T(r, f) .
\end{aligned}
$$

Similarly,

$$
\bar{N}\left(r, \frac{1}{G^{*}}\right) \leq 2 T(r, g) .
$$

Using Lemma 9, we have

$$
\begin{aligned}
& T\left(r, F^{*}\right)=(n+2) T(r, f)+S(r, f), \\
& T\left(r, G^{*}\right)=(n+2) T(r, g)+S(r, g) .
\end{aligned}
$$

Thus,

$$
\Theta\left(0, F^{*}\right)=1-\varlimsup_{r \rightarrow \infty} \frac{\bar{N}\left(r, 1 / F^{*}\right)}{T\left(r, F^{*}\right)} \geq 1-\frac{2}{n+2} .
$$

Similarly,

$$
\Theta\left(0, G^{*}\right) \geq 1-\frac{2}{n+2} .
$$

Therefore, (109) becomes

$$
\begin{aligned}
\Theta\left(0, F^{*}\right)+\Theta\left(c, F^{*}\right) & \geq 2\left(1-\frac{2}{n+2}\right) \\
& =\frac{2 n}{n+2} \geq \frac{14}{9}>1 \quad \text { for } n \geq 7,
\end{aligned}
$$

which contradicts $\sum_{a \in \mathscr{C}} \Theta(a, f) \leq 1$. Thus, we have

$$
\frac{f^{n+2}}{n+2}-\frac{f^{n+1}}{n+1}=\frac{g^{n+2}}{n+2}-\frac{g^{n+1}}{n+1} .
$$

Let $h=f / g$. If $h \neq \equiv 1$, then by (116) we have

$$
g=\frac{(n+2)\left(1+h+h^{2}+\cdots+h^{n}\right)}{(n+1)\left(1+h+h^{2}+\cdots+h^{n+1}\right)} .
$$

By Picard's theorem, $h(z)$ is a constant. Hence, $g$ is a constant, which is a contradiction. Therefore, $h(z) \equiv 1$, that is, $f(z) \equiv g(z)$.

\section{Remarks}

If the condition " $f^{n}(f-1) f^{\prime}$ and $g^{n}(g-1) g^{\prime}$ share $z$ CM" is replaced by the condition " $f^{n}(f-1) f^{\prime}$ and $g^{n}(g-1) g^{\prime}$ share $\alpha(z)$ CM," where $\alpha$ is a meromorphic function such that $\alpha \neq \equiv, \infty$ and $T(r, \alpha)=o\{T(r, f), T(r, g)\}$; the conclusion of Theorems 1, 2, and 3 still holds. We, thus, obtain the following results. 
Theorem 11. Let $f$ and $g$ be two nonconstant meromorphic functions, $n \geq 11$ a positive integer. If $f^{n}(f-1) f^{\prime}$ and $g^{n}(g-$ 1) $g^{\prime}$ share $\alpha(z) C M, f$ and $g$ share $\infty$ IM, then either $f(z) \equiv$ $g(z)$ or

$$
g=\frac{(n+2)\left(1-h^{n+1}\right)}{(n+1)\left(1-h^{n+2}\right)}, \quad f=\frac{(n+2) h\left(1-h^{n+1}\right)}{(n+1)\left(1-h^{n+2}\right)},
$$

where $h$ is a nonconstant meromorphic function.

Theorem 12. Let $f$ and $g$ be two nonconstant meromorphic functions, $n \geq 12$ a positive integer. If $f^{n}(f-1)^{2} f^{\prime}$ and $g^{n}(g-$ $1)^{2} g^{\prime}$ share $\alpha(z) C M$, $f$ and $g$ share $\infty I M$, then $f(z) \equiv g(z)$.

Theorem 13. Let $f$ and $g$ be two nonconstant entire functions, $n \geq 7$ an integer. If $f^{n}(f-1) f^{\prime}$ and $g^{n}(g-1) g^{\prime}$ share $\alpha(z) C M$, then $f(z) \equiv g(z)$.

\section{Acknowledgments}

The authors thank the referees for their valuable suggestions. This research work is supported by the Department of Science and Technology, Government of India, Ministry of Science and Technology, Technology Bhavan, New Delhi, India (no. SR/S4/MS: 520/08).

\section{References}

[1] C.-C. Yang and H.-X. Yi, Uniqueness Theory of Meromorphic Functions, vol. 557 of Mathematics and Its Applications, Kluwer Academic, Dordrecht, The Netherlands, 2003.

[2] L. Yang, Value Distribution Theory, Springer, Berlin, Germany, 1993.

[3] W. Lin and H. Yi, "Uniqueness theorems for meromorphic functions concerning fixed-points," Complex Variables, vol. 49, no. 11, pp. 793-806, 2004.

[4] J.-F. Xu, F. Lü, and H.-X. Yi, "Fixed-points and uniqueness of meromorphic functions," Computers and Mathematics with Applications, vol. 59, no. 1, pp. 9-17, 2010.

[5] C. C. Yang, "On deficiencies of differential polynomials. II," Mathematische Zeitschrift, vol. 125, pp. 107-112, 1972.

[6] H. X. Yi, "Meromorphic functions that share two or three values," Kodai Mathematical Journal, vol. 13, no. 3, pp. 363-372, 1990. 


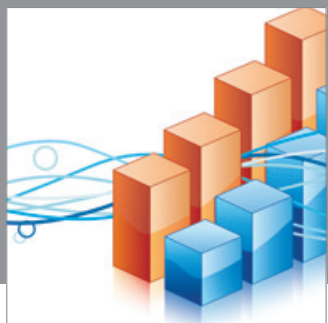

Advances in

Operations Research

mansans

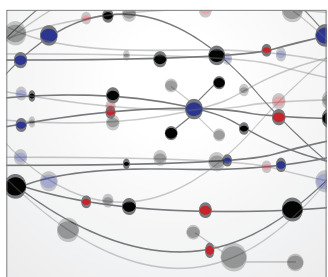

The Scientific World Journal
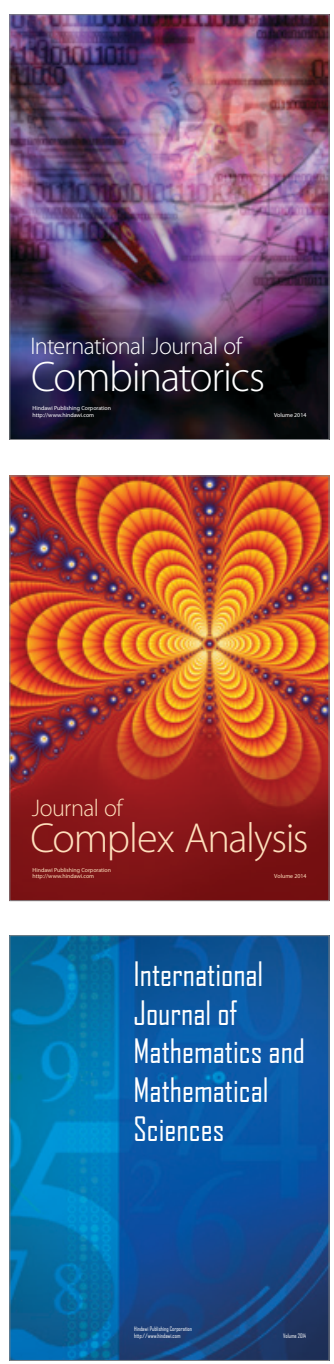
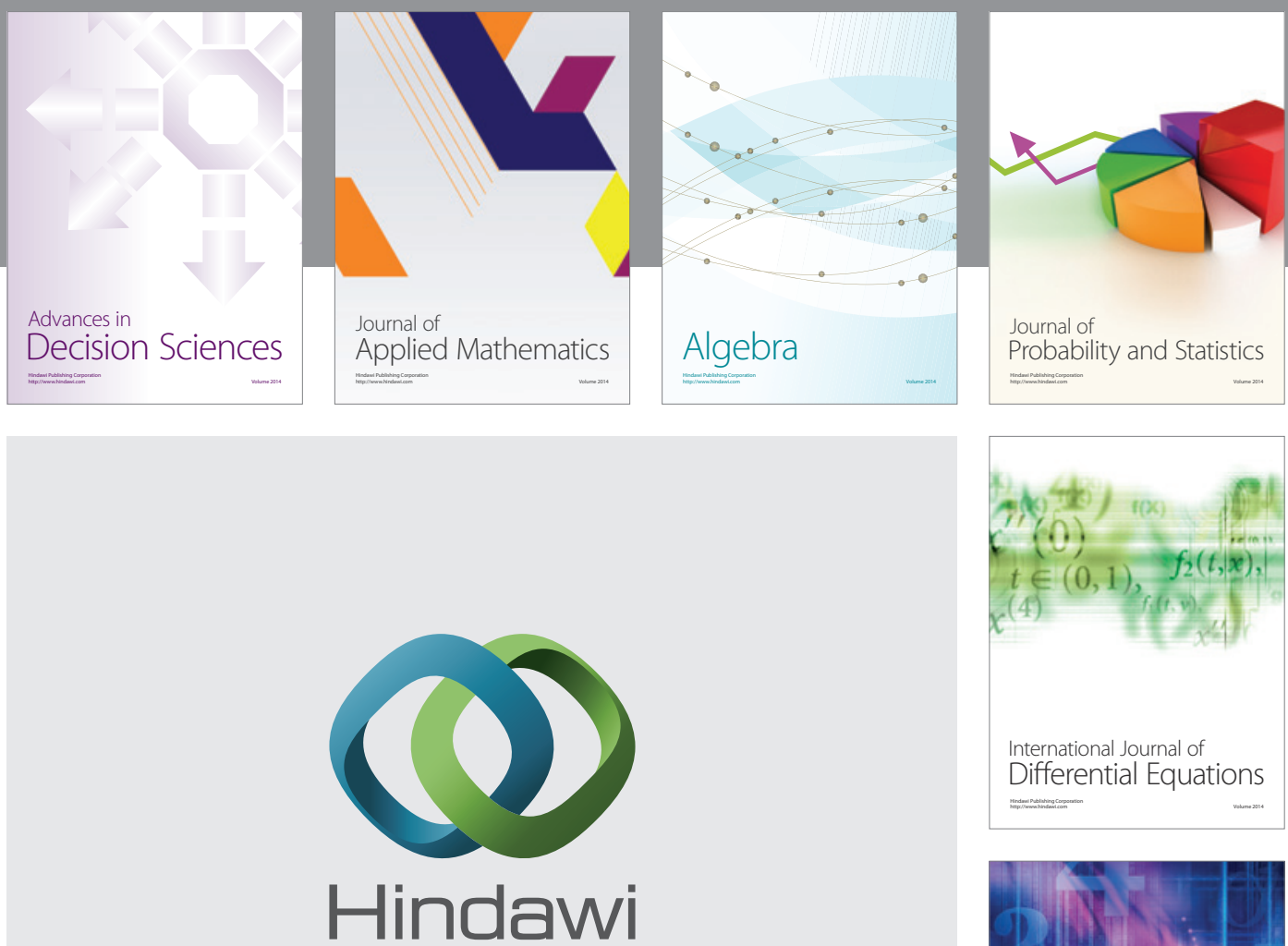

Submit your manuscripts at http://www.hindawi.com
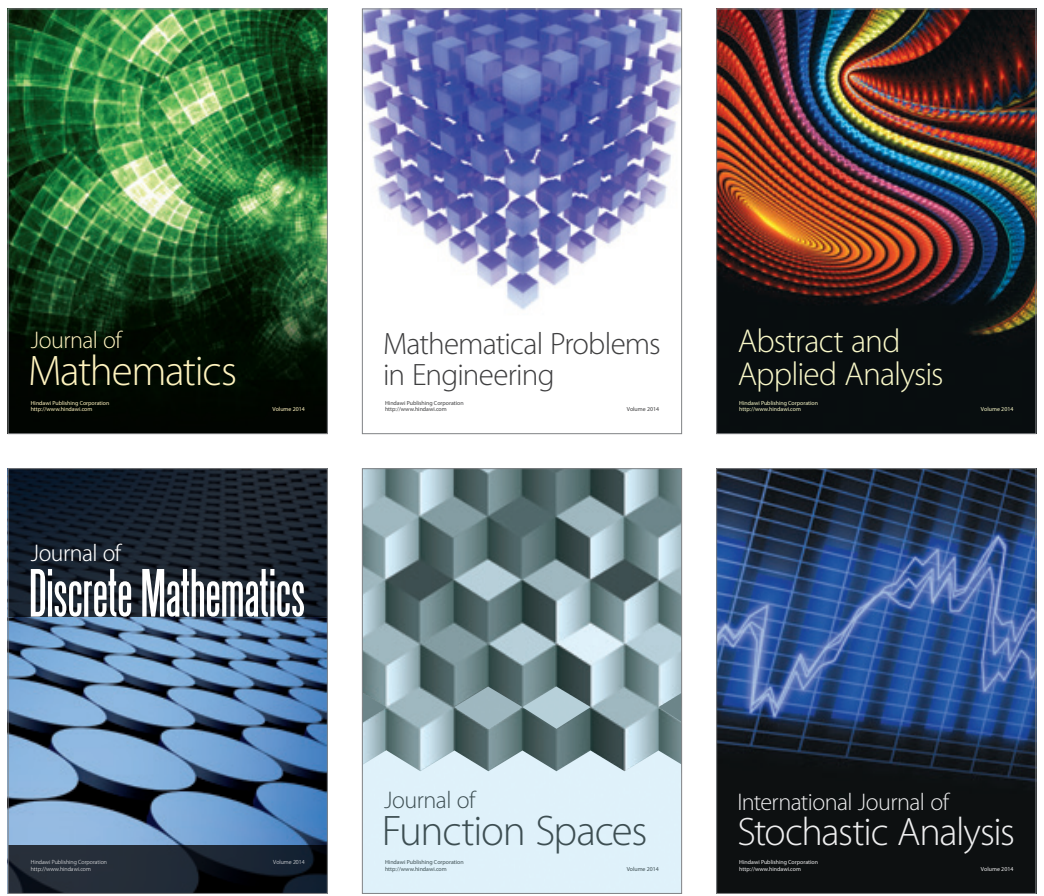

Journal of

Function Spaces

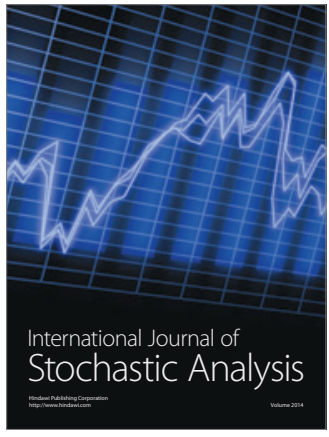

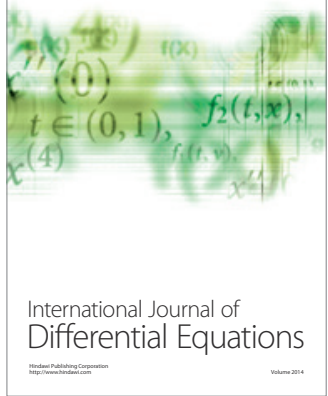
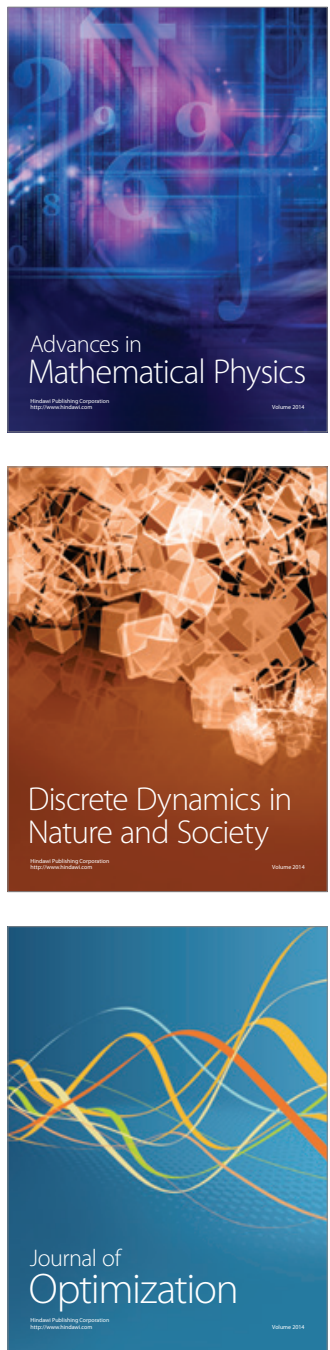